\title{
Edge Plasma Modelling for HT-7 Superconducting Tokamak Experiments
}

\author{
YiPing Chen ${ }^{a *}$, Guosheng $\mathrm{Xu}^{a}$, Jiansheng $\mathrm{Hu}^{a}$, D.P.Coster ${ }^{b}$ \\ ${ }^{a}$ Institute of Plasma Physics, Chinese Academy of Sciences, Hefei, Anhui, China \\ ${ }^{b}$ Max-Planck-Institut für Plasmaphysik, IPP-EURATOM Association, \\ Boltzmannstrasse 2, D-85748 Garching, Germany
}

\begin{abstract}
HT-7 is a medium-sized superconducting tokamak with limiter and circular cross section configuration and it has become an important target for modelling of experimental plasmas. SOLPS5.0(B2.5) edge plasma transport code is used for modelling the experimental shots in order to match the code results to the experimental temperature and density profiles by varying the particle transport coefficient $\mathrm{D}$ and the perpendicular heat conductivities $\chi_{e}, \chi_{i}$ in the code until good agreement between the code results and the measured data. For ohmic heated Deuterium plasma shot with toroidal magnetic field $B_{t}=1.98 \mathrm{~T}$, plasma current $I_{p}=140 \mathrm{kA}$, ohmic heating power $P=180 \mathrm{~kW}$, by matching the modelling results to the experimental profiles of electron density and temperature at the orthogonal plane, the perpendicular particle and heat transport coefficients $\mathrm{D}, \chi_{e}$ and $\chi_{i}$ are obtained from the modelling results in the edge computation region with the assumption of $\chi_{e}=\chi_{i}$.
\end{abstract}

PACS numbers: 52.25.Fi, 52.65.-y, 52.40.Hf, 52.55.Fa

Keywords: HT-7 tokamak, limiter, SOLPS5.0(B2.5), modelling

JNM keywords: Theory and Modelling, Plasma-Materials Interaction, Plasma Properties

PSI-17 Keywords: B2/EIRENE, Cross-Field Transport, Edge Modelling, Limiter

Corresponding author address: P.O.Box 1126, Hefei, Anhui, China

*Corresponding author E-mail: ypchen@ipp.ac.cn

Presenting author: YiPing Chen

Presenting author E-mail: ypchen@ipp.ac.cn 


\section{Introduction}

Edge plasma transport modelling codes are widely used for investigating specific physics questions in the edge plasma of tokamaks. On transport time-scales, a fluid representation is generally used for the edge plasma and a set of two dimensional fluid transport equations dealing with the density, momentum and energy for each plasma species usually are solved in the codes to determine the magnitudes and the profiles of the plasma parameters such as density, temperature. The two dimensional thansport equations include parallel and perpendicular transport coefficients respectively, the parallel transport coefficients usually are calculated from the neoclassical formulation with flux limiting and the perpendicular transport coefficients usually are decided by the perpendicular anomalous transport coefficients. Due to the uncertainties associated with the perpendicular anomalous particle transport coefficient D, the perpendicular anomalous energy transport coefficients $\chi_{e}$ and $\chi_{i}$, some assumption for the perpendicular transport coefficients usually is made according to a reasonable judgement. In ITER divertor modelling by using B2-Eirene[1,2], the cross-field transport coefficients are assumed to be constant with $\chi_{e}=\chi_{i}=1 \mathrm{~m}^{2} / \mathrm{s}, D=0.3 \mathrm{~m}^{2} / \mathrm{s}$, these values results in a typical width of the power load profile on the midplane $\lambda_{q}=0.5 \mathrm{~cm}$, and in a radial pressure gradient in the midplane close to experimental ballooning limit[3].

For modelling experimental plasmas by using a transport code, the perpendicular transport coefficients in the code usually are varied until good agreement between the code results and the measured data, so, the perpendicular transport coefficients can be obtained by fitting the code results to the experiment measurement data. OSM2/EIRENE[4] and EDGE2D[5] have been used for modelling JET shots and the experimental profiles are matched by the codes results. Interpretative modelling of the SOL palsma in ASDEX Upgrade has been carried out by using SOLPS5.0[1,2,6] for profiles determination of the cross-field transport coefficients[7]. In present work the SOLPS5.0 is used for modelling experimental plasma in HT-7 limiter tokamak and the perpendicular transport coefficients have been 
obtained by fitting the SOLPS5.0 results to the experimental profiles of plasma temperature and density at the orthogonal plane in the experimental shot.

\section{The modelling of the experimental shot in HT-7 tokamak}

HT-7 is a medium-sized superconducting tokamak with limiter and circular cross section configuration, the main parameters of the device are $R=122 \mathrm{~cm}, a=27 \mathrm{~cm}, I_{p}=100-225 \mathrm{kA}, B_{t}=1.5-2 \mathrm{~T}$. The limiter consists of two poloidal limiters and a toroidal belt limiter, the toroidal belt limiter with height of $120 \mathrm{~mm}$ is located at HFS, the two poloidal limiters locate respectively at the south and the north of the torus, each one has toroidal width of $120 \mathrm{~mm}$. The poloidal limiters form two circles respectively at the south and the north of the torus, each circle includes a small part of the toroidal belt limiter at the HFS. The structure view of HT-7 limiters is shown in Fig.1. SOLPS5.0(B2.5) is a two dimentional fluid transport code for tokamak edge plasma modelling. The two dimentional modelling for edge plasmas in HT-7 tokamak by using SOLPS5.0(B2.5) mainly handle the domain covered by the poloidal limiter which has $120 \mathrm{~mm}$ extension in the toroidal direction. If the poloidal limiter has full extension in the toroidal direction, the domain covered by the limiter has toroidally symmetry, the two dimentional modelling can exactly handle the domain, but, in present geometry structure of HT-7 tokamak the poloidal limiter has only $120 \mathrm{~mm}$ extension in the toroidal direction, the two dimentional modelling is only a very rough approximation for this real three dimentional geometry.

The modelling of experimental plasma is carried out by modelling the ohmicly heated deuterium plasma shot. In the shot, toroidal magnetic field $B_{t}=1.98 T$, plasma current $I_{p}=140 \mathrm{k}$, loop voltage $V_{p}=1.3 \mathrm{~V}$, the ohmic heating power $P=180 \mathrm{~kW}$, centre chord-averaged electron density $n_{e}=1.5 \times 10^{19} / \mathrm{m}^{3}$. The plasma is circular cross section configuration and the position of plasma centre in the shot locates at $R \sim 122+1.3 \mathrm{~cm}, Z \sim-0.5 \mathrm{~cm}$. The SOLPS5.0(B2.5) modelling needs MHD equilibrium data for the computation mesh production, in the shot the real MHD equilibrium is replaced 
by pre-producd MHD equilibrium with circular cross section configuration from an equilibrium code due to lack of MHD equilibrium reconstruction data in the shot and the experiment data of the total plasma current is used for magnetic field computation at the computational mesh points. The position of plasma centre is shifted to $R=122+1.3 \mathrm{~cm}, Z=-0.5 \mathrm{~cm}$ in the modelling for matching plasma position in the shot. The computational meshes are produced by using Carre code in the SOLPS5.0 code package based on the MHD equilibrium data, the real geometry of limiter and other components in the device are taken into account. The computational meshes produced by Carre is shown in Fig.2.

In previous modelling for limiter tokamaks, for example TEXTOR, by using B2 code[8,9], Carre is not applied to the computation mesh production, the computational meshes are generated by an analytic method. With the analytic method the conformal transformation is used, by choosing the free parameters according to the limiter geometry in the device the computational meshes can be generated. Due to B2 calculates on an orthogonal mesh and plasma does not flow to inclined surfaces, so, when the cicular magnetic flux surfaces and the striking surface at the limiter are orthogonal the orthogonal curvilinear co-ordinate meshes for B2 modelling calculation can be produced easily by the analytic method. In present SOLPS5.0(B2.5) modelling for HT-7 tokamak, the plasma striking surface at the poloidal limiter is arc and the surface does not orthogonalize the flux surfaces, so, it is very difficult to produce calculational meshes by the analytic method. With the development of SOLPS5.0, now it is possible to model edge plasma in a limiter tokamak with all kinds of limiter, such as poloidal or toroidal limiter, Carre can be used for the mesh production and the real geometry of limiter and components in the device can be taken into account, a more precise modelling results in the computation region near the separatrix striking point at the limiter can be obtained.

The boundary conditions are reasonably set for present SOLPS5.0(B2.5) modelling. At the coreSOL boundary the energy flux from the boundary to the computational region is set to be $P=180 \mathrm{~kW}$ with the assumption that the electron heat flux $P_{e}$ is equal to the ion heat flux $P_{i}$, i.e. $P_{e}=P_{i}=90 \mathrm{~kW}$. The sheath boundary condition is set at the limiter striking surface. The pumping is set at the north 
boundary, the outer wall, with the particle leakage $\Gamma_{\text {loss }}=\alpha c_{s} n_{e}, \alpha=0.01$ for neutral and $\alpha=0.001$ for ion. The plasma species include the deuterium neutral and deuterium ion, the impurity species are not included. The drift terms in the fluid equations are switched off.

The experimental profiles of the electron density and temperature at the orthogonal plane are shown in Fig.3. From Fig.3 the range of the profiles in the computational region can be divided into two sections, the first one, section A, which includes mainly core plasma, is the part from $\mathrm{r}-\mathrm{a}=0.02 \mathrm{~m}$ to the separatrix $\mathrm{r}-\mathrm{a}=0$, in section $\mathrm{A}$ the profile curves are steeper. The second one, section $\mathrm{B}$, which includes SOL plasma, is the part from separatrix $r-a=0$ to $r-a=0.02 m$, in section $B$ the profile curves become flater. From $\mathrm{r}-\mathrm{a}=0.02 \mathrm{~m}$ to $\mathrm{r}-\mathrm{a}=0.04 \mathrm{~m}$, the profile curves become steeper again, in the last section, from $\mathrm{r}-\mathrm{a}=0.04 \mathrm{~m}$ to the end, the profile curves become flater than in section B. The computational region includes only sections $\mathrm{A}$ and $\mathrm{B}$. It can be expected that the radial across-field transport coefficients are different between section A and section B. So, the different radial across-field transport coefficients should be taken into account for the SOLPS5.0 modelling in order to match the code results to the experimental profiles in the sections A and B. The cross-field particle transport coefficient D and the cross-field heat transport coefficients $\chi_{e}, \chi_{i}$ are varying in the sections A and B respectively until good agreement between the profiles from the code results and the experimental profiles shown in Fig.3. The matching results are shown in Fig.3 and the profiles of D, $\chi_{e}$ and $\chi_{i}$ obtained from the matching are shown in Fig.4. The $\mathrm{D}=1.15 \mathrm{~m}^{2} / \mathrm{s}, \chi_{e}=\chi_{i}=8.0 \mathrm{~m}^{2} / \mathrm{s}$ in the section $\mathrm{A}$ and $\mathrm{D}=1.38 \mathrm{~m}^{2} / \mathrm{s}$, $\chi_{e}=\chi_{i}=16.0 \mathrm{~m}^{2} / \mathrm{s}$ in the section $\mathrm{B}$ can be found from Fig.4. The matching results also show the maximum electron temperature at the limiter is $T_{e}=37.56 \mathrm{eV}$, the pumping speed $S_{p}=4.12 \times 10^{20} \mathrm{D} / \mathrm{s}$.

The plasma energy flux at the separatrix striking point at the limiter is one of key issues, it is associated with the radial transport coefficients $\mathrm{D}, \chi_{e}$ and $\chi_{i}$. With the radial transport coefficients $\mathrm{D}$, $\chi_{e}$ and $\chi_{i}$ obtained from the matching the parallel electron and ion energy fluxes near the separatrix striking point at the limiter are showed respectively by Fig.5 and Fig.6. The electron energy flux with the maximum value of $q_{e}=21.70 \mathrm{~kW} / \mathrm{m}^{2}$ is much larger than the ion energy flux with the maximum 
value of $q_{i}=0.15 \mathrm{~kW} / \mathrm{m}^{2}$. The maximum values of $q_{e}$ and $q_{i}$ locat at the separatrix striking point at the limiter. The temperature rise $T(x, t)$ due to heat source $q(t)$ can be expressed as

$$
T(x, t)=\frac{1}{\rho C} \int_{0}^{t} \frac{q(t)}{\sqrt{4 \pi \alpha t}} \exp \left(-\frac{x^{2}}{4 \alpha t}\right) d t
$$

$\rho, \mathrm{C}$ and $\alpha$ are respectively the density of limiter material, specific heat at constant volume and heat diffusivity, $\alpha=\kappa / \rho C, \kappa$ is thermal conductivity. $q(t)$ is energy flux at time $\mathrm{t}, T(x, t)$ is the temperature rise at time $t$ and at the distance $x$ under the separatrix striking point at the limiter. The limiter is made of Carbon, the $\rho=1.87 \times 10^{3} \mathrm{~kg} / \mathrm{m}^{3}, C=1300 \mathrm{~J} / \mathrm{kg} . \mathrm{k}$, thermal conducitivity $\kappa=118 \mathrm{~W} / \mathrm{m} . \mathrm{k}$, which leads to $\alpha=4.85 \times 10^{-5} \mathrm{~m}^{2} / \mathrm{s}$. At maximum heat flux $q(t)=q=q_{e}+q_{i}=21.85 \mathrm{~kW} / \mathrm{m}^{2}$, the temperature rise $T(x, t)$ at $x=3 \mathrm{~mm}$ and $t=60 \mathrm{~s}$ is only $5.36^{\circ} \mathrm{C}$. The temperature rise $T(x, t)=5.36^{0} \mathrm{C}$ is not high duo to low plasma energy flux $q$ at the separatrix striking point at the limiter and the temperature rise $T(x, t)$ is not high enough to cause the limiter material damaged seriously. But, with the movement of plasma centre position towards the striking surface at the limiter and the increase of heating power, the $q$ can rise to higher values, if $q(t)=q=1 M W / m^{2}$, the temperature rise $T(x, t)$ at $x=3 m m$ and $\mathrm{t}=60 \mathrm{~s}$ is $245.5^{0} \mathrm{C}$ according to Eq.(1).

\section{Conclusions}

SOLPS5.0 is used for modelling experimental plasma shot in HT-7 limiter tokamak and the experimental profiles of plasma density and temperature are fitted by the code results in order to get the cross-field particle transport coefficient D and heat transport coefficients $\chi_{e}, \chi_{i}$. The real limiter geometry is taken into account by using Carre mesh production code in the modelling. The modelling and fitting results show that the cross-field transport coefficients are different between the section within the separatrix and the section outer of the separatrix. The cross-field transport coefficients are larger in the section outer of the separatrix than in the section within the separatrix. The maximum value 
of total plasma energy flux at the separatrix striking point on the limiter in the shot is $21.85 \mathrm{~kW} / \mathrm{m}^{2}$ which causes the temperature rise at the position $x=3 \mathrm{~mm}$ under the separatrix striking point is about $5.36^{0} \mathrm{C}$.

\section{Acknowledgments}

This work was supported by National Natural Science Foundation of China (No.10275066) and supported partially by the JSPS-CAS Core-University Program in the field of "Plasma and Nuclear Fusion".

\section{References}

[1] D.P.Coster, X.Bonnin, et al., J. Nucl. Materials, 337-339(2005)366-370.

[2] D.Reiter, J. Nucl. Materials, 196-198(1992)80.

[3] A.S.Kukushkin, H.D.Pacher, G.W.Pacher, G.Janeschitz, Nucl. Fusion 43(2003)716-723.

[4] W Fundamenski, et al., Plasma Phys. Control. Fusion, 44(2002)761-793.

[5] A Kallenbach, Y Andrew, et al., Plasma Phys. Control. Fusion 46(2004) 431-446.

[6] V.A.Rozhansky, S.P.Voskoboynikov, E.G.Kaveeva, D.P.Coster, R.Schneider, Nucl. Fusion, Vol.41, No.4(2001) $387-401$.

[7] L.D. Horton, A.V. Chankin, Y. P. Chen, G.D.Conway, D.P.Coster et al., Nucl. Fusion 45(2005)856-862.

[8] M. Baelmans, Code Improvements and Applications of a Two-dimensional Edge Plasma Mode for Toroidal Devices, Ph.D. thesis, Katholieke Universiteit Leuven(Belgium),1994.

[9] D.S.Gray, M.Baelmans, J.A.Boedo, D.Reiter and R.W.Conn, Physics of Plasma, Volume 6, No.7(1999)2816. 
Figure Captions:

Fig.1 Basic structure view of HT-7 limiter.

Fig.2 The computation meshes for edge plasma modelling in HT-7 limiter tokamak by using SOLPS5.0(B2.5).

Fig.3 The fitting results of HT-7 experimental data.

Fig.4 The D and $\chi_{e}$ profiles obtained by fitting experimental profiles of HT-7 tokamak.

Fig.5 Parallel electron energy flux from the modelling.

Fig.6 Parallel ion energy flux from the modelling. 
HT-7 tokamak limiter

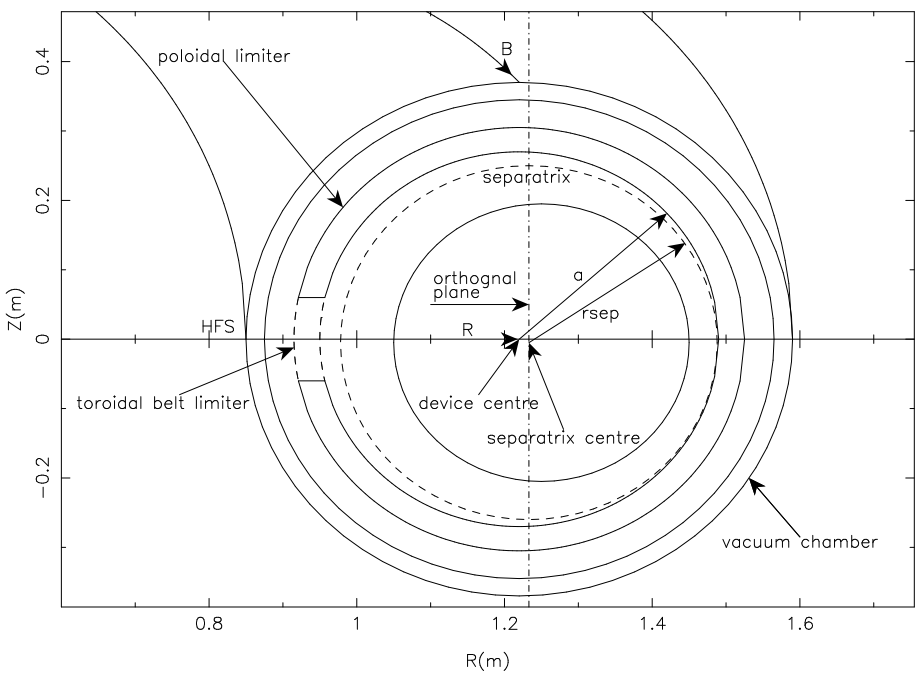

Fig.1 


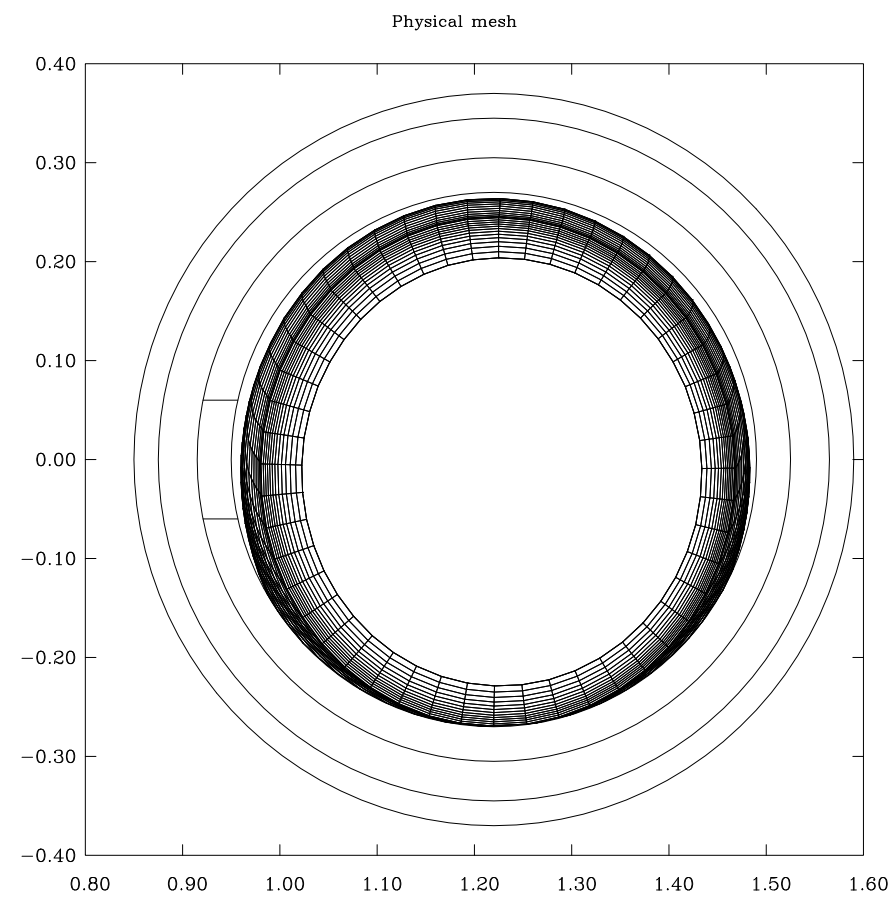

Fig.2 


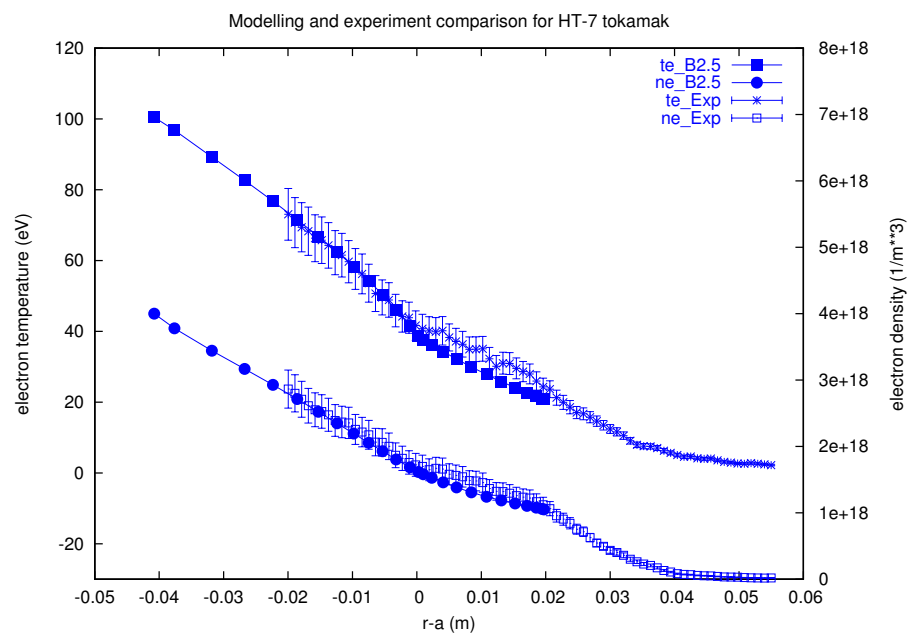

Fig.3 


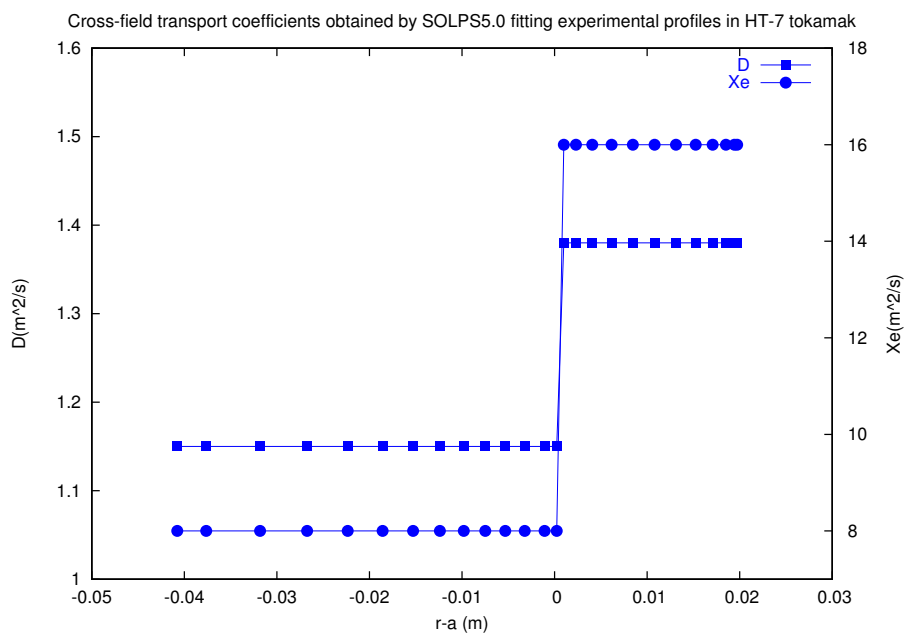

Fig.4 


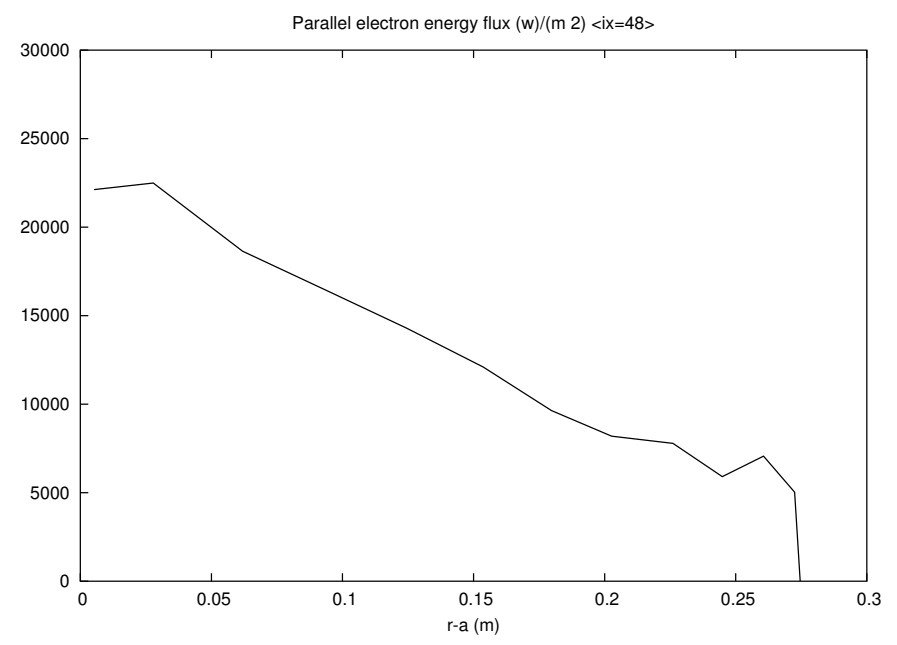

Fig.5 


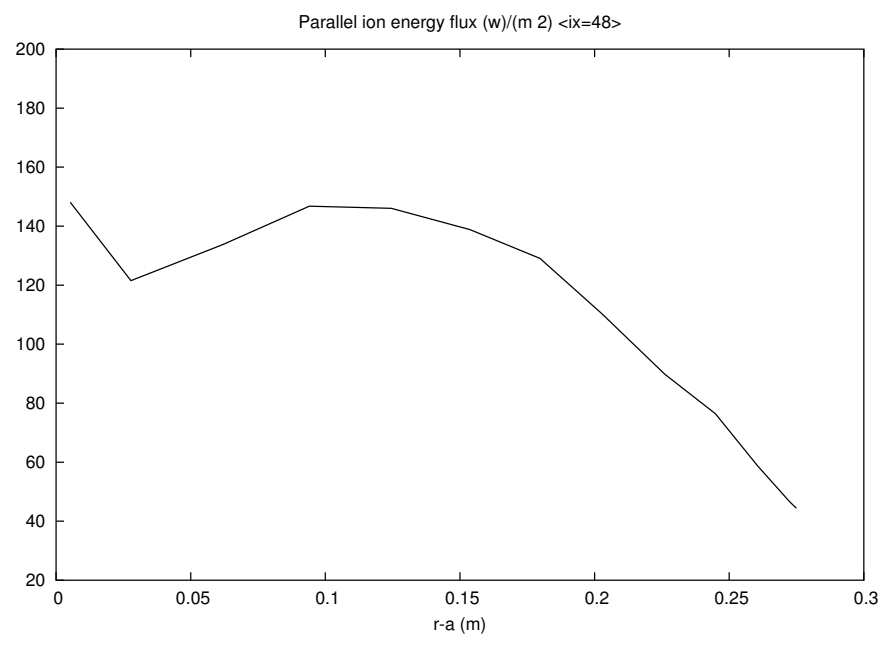

Fig.6 